Available online at:

https://acta-acustica.edpsciences.org

\title{
A notched-noise precursor affects both diotic and dichotic notched-noise masking
}

\author{
Felix Dymel ${ }^{1}$, Monika Kordus ${ }^{1}$, Ifat Yasin $^{2}$, and Jesko L. Verhey ${ }^{1, *}$ (B) \\ ${ }^{1}$ Department of Experimental Audiology, Otto von Guericke University, Leipziger Str. 44, 39120 Magdeburg, Germany \\ ${ }^{2}$ Department of Computer Science, University College London, Gower Street, London WC1E 6EA, United Kingdom
}

Received 18 November 2020, Accepted 2 September 2021

\begin{abstract}
The present study investigates how diotic and dichotic masked thresholds, in a notched-noise masking paradigm, are affected by activation of the Medial OlivoCochlear (MOC) reflex. Thresholds were obtained for a $500-\mathrm{Hz}$ pure tone diotic or a dichotic signal, $S$ ( $S_{0}$ or $S_{\pi}$ respectively), in the presence of a simultaneous or forward diotic masker (bandpass noise with no notch or a $400-\mathrm{Hz}$ notch). A diotic precursor sound (bandpass noise with a 400- or $800-\mathrm{Hz}$ notch) was presented prior to the signal and masker to activate the MOC reflex. For simultaneous- and forward-masking conditions, the decrease in masked thresholds as a notch was introduced in the masker was larger for the diotic than for the dichotic condition. This resulted in a reduced binaural masking level difference (BMLD) for the masker with a notch. The precursor augmented these two effects. The results indicate that the effect of the precursor, eliciting the MOC reflex, is less pronounced when binaural cues are processed.
\end{abstract}

Keywords: Binaural, Masking, Suppression, Auditory frequency selectivity

\section{Introduction}

Two physiological processes operate in normal hearing $(\mathrm{NH})$ individuals to improve speech perception in noise. One is binaural unmasking enabling the speech signal of interest to be enhanced relative to the background noise. The second process involves a brainstem-mediated efferent neural pathway [Medial OlivoCochlear (MOC) reflex], which modifies the cochlear response to sound [1]. Studies have often investigated binaural processing or neural feedback separately from one another, i.e., investigated the effect of neural feedback on monaural processing or investigated binaural-cue processing without considering neural feedback. In humans, the effect of the MOC reflex on monaural hearing has been studied using otoacoustic emissions (OAE) or psychoacoustics; both techniques show that the MOC reflex reduces cochlear gain, compression [2], and monaural frequency selectivity [3]. Binaural processing has been studied (by measuring for instance, $S_{0}$ and $S_{\pi}$ detectability in $N_{0}$ ) but without explicitly testing for the effects of the MOC reflex. The main aim of this study is to measure the contribution of the MOC reflex on estimates of frequency selectivity in binaural hearing. In particular, this study aims to answer the question as to whether the effects of the MOC reflex on the frequency selectivity of monaural and binaural auditory processing are equivalent

*Corresponding author: jesko.verhey@med.ovgu.de or if there is an effect of the MOC reflex that is specific to the binaural auditory system.

The cochlea is a mandatory stage of the peripheral auditory pathway. Its mechanical properties enable the auditory system to analyse the spectrum of a sound by a frequency-place transformation within the cochlea. In the living cochlea, active processes are thought to be responsible for a sharpening of the frequency tuning along the cochlea, as well as an amplification of the excitation at low-tomedium sound levels, resulting in cochlear gain [4].

These processes are commonly related to outer hair cell (OHC) activity [5]. Neural feedback (such as the MOC reflex) from higher stages of the auditory system can alter this response at the cochlear level. The MOC reflex includes an efferent pathway, originating in the brainstem, with connections in the MOC system, that innervates the cochlea of the inner ear $[6,7]$. Efferent projections to the cochlea make synaptic contact directly at the base of the OHCs, and efferent activation due to the MOC reflex leads to a modulation of the response of the basilar membrane (BM) to sound [8], specifically a reduction of the cochlear response to sound $[1,9]$. Several studies suggest that the MOC reflex improves the perception of an auditory signal, or even speech, in a noisy background [10-12].

Psychophysical $[2,13,14]$ and OAE $[15,16]$ studies in humans, measuring the effects of the MOC on signal detectability, have often used a precursor sound (or an inducer/elicitor sound) to elicit activation of the MOC. 
Psychophysical studies indicate that introduction of a precursor sound (to activate the MOC reflex) presented prior to the signal of interest (most often monaural) can affect cochlear gain, compression, as well frequency selectivity $[2,13,14,17]$. Yasin et al. [2] showed psychoacoustic data that were consistent with the interpretation that activation of the MOC reflex by presentation of a precursor ( $\geq 40 \mathrm{~dB} \mathrm{SPL}$ ) prior to the signal to be detected (hereafter simply referred to as the signal) resulted in a decrease in maximum gain and compression, with a linearization of the compressive function for precursor levels between 50 and $70 \mathrm{~dB}$ SPL.

Earlier masking studies of binaural hearing did not specifically use a "precursor sound", but masker fringes preceding the signal of interest (also referred to as forward noise fringes) which could have elicited the MOC reflex and affected the subsequent interpretation of results. For instance, McFadden [18] used simultaneous masking and showed that signal detectability in the $\mathrm{N}_{0} \mathrm{~S}_{\pi}$ condition decreased when noise and signal onset and offsets were coincident but improved when the noise onset preceded onset of the signal "forward noise fringe". Improved signal detectability in simultaneous masking noise for the $\mathrm{N}_{0} \mathrm{~S}_{\pi}$ condition (compared to the $\mathrm{N}_{0} \mathrm{~S}_{0}$ condition) has also been shown by Yost [19] and Gilkey et al. [20]. Yasin and Henning [21] looked at the effects of forward and backward fringe effects on signal detectability and the binaural masking level difference. The Yasin and Henning [21] study suggested that the effects of a noise fringe preceding a signal and simultaneous masker involved a complex combination of across-time, and across-filter listening strategies, as well as gain reduction due to the forward fringe (akin to the precursor used in the current binaural experiment). Other studies have also investigated the effect on binaural detectability with longduration $\mathrm{N}_{0}$ maskers [masker components either preceding (forward fringe) or extending after (backward fringe) the signal presentation] (e.g., [19, 20, 22-28]). In some instances, the $\mathrm{N}_{0}$ masker components may have activated the MOC reflex (although this idea was not explicitly tested in these studies). Current binaural models (e.g., [29, 30]) are unable to fully account for the effects of the MOC reflex on binaural cue processing and efferent effects are likely to contribute to binaural processing in complex ways $[3,21]$.

With regards to the effect of a precursor on estimates of frequency selectivity, both physiological [16] and psychophysical [31] studies show that gain reduction due to direct electrical stimulation/precursor presentation reduces monaural cochlear frequency selectivity. Psychoacoustic studies to investigate the frequency selectivity of the human ear have used a range of masking paradigms. A measure of frequency selectivity has often been obtained by measuring detectability of a signal whilst increasing the bandwidth of the masker [32]. In such an experiment, thresholds increase as masker bandwidth increases up to a certain critical bandwidth; a bandwidth determined by the energy falling within the auditory filter passband. Another methodology often used to measure frequency selectivity is one in which the detectability of a signal is measured in the presence of a masker with a spectral notch [33-35]. The notch-width is the parameter that is manipulated, and the rate of fall of the threshold as the notch-width is widened, provides an estimate of the filter bandwidth. For both the masker band-widening and the notched-noise experiments, the estimate of the auditory filter bandwidth centred at the signal frequency (with diotic $\mathrm{N}_{0}$ noise) depends on the binaural signal phase presented with the noise, i.e. $\mathrm{N}_{0} \mathrm{~S}_{0}$ or $\mathrm{N}_{0} \mathrm{~S}_{\pi}$ (for a review, see [36]).

A recent study by Verhey et al. [3] combined psychoacoustical methods traditionally used to analyze monaural gain-reduction effects, with methods used to investigate binaural frequency selectivity in normal-hearing listeners, for a $1000-\mathrm{Hz}$ signal. Thresholds for an $\mathrm{S}_{0}$ and $\mathrm{S}_{\pi}$ signal were measured in the presence of a simultaneous $25-\mathrm{ms}$ diotic $\mathrm{N}_{0}$ notched-noise masker for various notch widths. The effect of a precursor on the frequency selectivity derived from the notched-noise data was examined by presenting a broadband precursor (325-ms, $63 \mathrm{~dB}$ SPL) prior to the masker-signal stimulus. The addition of a precursor effectively reduced the frequency selectivity, supporting the hypothesis that cochlear gain is reduced due to the presence of the precursor. The size of the effect differed between the $\mathrm{N}_{0} \mathrm{~S}_{0}$ and $\mathrm{N}_{0} \mathrm{~S}_{\pi}$ conditions, indicating that there may be a specific effect of the MOC reflex on the binaural auditory system.

Unfortunately, the maximum difference between the diotic and dichotic conditions was only a few decibels, which may have been partly due to the choice of signal frequency $(1 \mathrm{kHz})$ and the short duration $(25 \mathrm{~ms})$ of the signal. Furthermore, since a simultaneous-masking paradigm was used, it is difficult to disentangle cochlear suppressive effects from the overall masking effects, which would have influenced subsequent estimates of cochlear frequency selectivity. In the latter case, stimulus choice as well as presentation paradigm may have contributed to the reduced effect of the precursor on binaural processing shown by Verhey et al. [3].

To overcome this problem, the present study uses a forward-masking paradigm, (in which the signal is not suppressed by the masker) as well as a simultaneous-masking paradigm, to facilitate a comparison with the results of Verhey et al. [3]. Binaural filter widths are often estimated to be wider than monaural filter widths (e.g. [37], for a review, see [36]), and the ratio of the binaural to monaural filter width seems to remain unchanged with a hearing impairment [38]. If a precursor acts to reduce gain applied to a signal then it would be expected to do so to a similar degree irrespective of the signal's interaural phase. As mentioned earlier, the data in [3] indicated that this may not be the case, i.e., that the influence of the precursor is different for the diotic and dichotic condition. However, this could just be a consequence of the experimental design used in [3]. To further investigate the effect of eliciting the MOC reflex on monaural and binaural processing, the present study extends the findings of [3] by using a signal frequency of $500 \mathrm{~Hz}$ (rather than $1 \mathrm{kHz}$ ) to facilitate a larger binaural masking level difference. The present study also differs from [3] with regards to the spectral characteristics of the precursor. Verhey et al. [3] used a broadband-noise 
precursor which contributed to some forward masking of the subsequently-presented stimuli. The present study attempts to reduce this forward masking effect by introducing a spectral notch in the precursor stimulus, centred at the signal frequency. We hypothesize that using an experimental design in which the (i) forward masking due to the precursor are reduced and (ii) a signal frequency of $500-\mathrm{Hz}$ is used to increase the binaural masking level difference, the precursor will exert an equivalent effect on gain reduction and hence frequency selectivity measures in both diotic and dichotic signal conditions.

\section{Materials and methods}

Thirteen listeners (nine female, four male), aged between 21 and 40 years, participated in the experiment. Three listeners were unpaid volunteers of the research team and ten listeners were paid volunteers. All listeners had normal hearing within a frequency range from 0.125 to $8 \mathrm{kHz}[<15 \mathrm{~dB}$ hearing level $(\mathrm{HL})]$. The research was approved by the Ethics committee at the Medical Faculty of the University of Magdeburg and was conducted according to the principles expressed in the Declaration of Helsinki.

Figure 1 shows the envelopes (first row) and spectra (second to fourth rows) of the different masking conditions. The signal (thick black line in second to fourth rows) was a $500-\mathrm{Hz}$ pure tone with a duration of $10 \mathrm{~ms}$, including 5 -ms raised-cosine ramps at the onset and offset. The signal was either presented in phase to the two ears $\left(\mathrm{S}_{0}\right)$ or had an interaural phase difference of $\pi$ between the right and the left ear $\left(\mathrm{S}_{\pi}\right)$. Thresholds of the signal were measured in quiet, and in the presence of a notched-noise masker.

The masker was a 30-ms long bandpass-filtered noise (60-2000 Hz) with a spectral notch arithmetically centred at the signal frequency. The masker was always presented diotically $\left(\mathrm{N}_{0}\right)$. The rectangles with left-sloping stripes in the 2 nd to 4 th row depict the masker without a notch. In addition to this no-notch masker, a masker with a notch width of $400 \mathrm{~Hz}$ was used. The spectral edges of this notch are indicated by dashed horizontal lines. The masker spectrum level was $30 \mathrm{~dB}$. Thus, the overall level of the bandpass-filtered noise without a notch was $63 \mathrm{~dB}$ SPL. The masker was generated by transforming a Gaussian white noise of the desired duration into the frequency domain via a Fast Fourier Transform (FFT), setting all Fourier components outside the desired passband (60-2000 Hz), as well as those within the notch, to zero and performing an inverse FFT on the complex spectrum. The resultant noise waveforms were then gated on and off with 5-ms raised cosine. The short duration of the masker-signal-complex was chosen to avoid self-activation of the MOC reflex [16].

Thresholds were measured for the signal embedded in the masker alone [referred to as the no-precursor (NoP) condition] and in the presence of a precursor presented prior to the masker. Parameters of the precursor were chosen to elicit a large gain reduction (and hence noticeable changes in frequency selectivity measures) on the basis of the findings of [5]. The precursor was a bandpass-filtered noise $(60-2000 \mathrm{~Hz})$ with a spectral notch at the signal frequency, to reduce forward masking. The notch width was either $400 \mathrm{~Hz}$ or $800 \mathrm{~Hz}$. Precursors with a 400-Hz or $800-\mathrm{Hz}$ notch are abbreviated as NNP4 and NNP8, respectively. Their temporal and spectral characteristics are schematically shown, in the second and third rows of Figure 1, as rectangles with right-sloping stripes. The precursor duration was $325 \mathrm{~ms}$, including 10-ms raised-cosine ramps at on- and offset. The precursor had the same spectrum level as the masker $(30 \mathrm{~dB})$. The silent interval between precursor and signal was $55 \mathrm{~ms}$ long. This duration was chosen to further reduce the forward-masking effect on the signal due to the precursor, whilst still eliciting a substantial gain-reduction effect when processing masker and signal. To determine the effect of any forward masking due to the precursor on the subsequent signal, thresholds were also obtained for the signal presented in the presence of only the precursor (in the absence of the masker).

The experiment comprised of 18 conditions [ 3 precursors $(\mathrm{NoP}, \mathrm{NNP} 4$, and NNP8) $\times 3$ masking conditions (no notch, $400-\mathrm{Hz}$ notch, and no masker $) \times 2$ binaural conditions $\left(S_{0}\right.$ and $\left.\mathrm{S}_{\pi}\right)$ ]. For each condition, thresholds for the signal were determined with a three-interval three-alternative forced-choice procedure. The 400-ms stimulus intervals of a trial were separated by silence periods of $400 \mathrm{~ms}$. One randomly selected interval included the signal. The signal always started $380 \mathrm{~ms}$ after interval onset. The same onset of the signal was used in all conditions to ensure that the possible effect of forward masking due to the precursor is the same for both masking conditions.

In the case of the masking conditions, a masker was present in all intervals of a trial. The masker onset was either $350 \mathrm{~ms}$ or $370 \mathrm{~ms}$ after interval onset. For the onset at $350 \mathrm{~ms}$, the offset of the masker coincided with the onset of the signal (forward-masking condition). For the masker onset at $370 \mathrm{~ms}$, the signal was temporally centred in the masker (simultaneous-masking condition). In the precursor conditions, the precursor was present in all intervals of a trial. The precursor started at the beginning of an interval. Random noise was used, i.e., for each presentation of the precursor and of the masker a new noise was generated.

The task of the listener was to indicate the interval containing the signal by pressing the corresponding button on the keyboard. The initial signal level was set at $85 \mathrm{~dB}$ SPL, i.e., well above threshold. An adaptive procedure with a one-up two-down rule was used, i.e., signal level was reduced after two consecutive correct responses and increased after one false response. The initial step-size was $8 \mathrm{~dB}$. The step size was halved after each upper reversal until the minimum step-size of $1 \mathrm{~dB}$ was reached. For this minimum step size, the run continued for six reversals. The average of the signal levels at these six reversals was taken as the threshold estimate. For each condition, this procedure was repeated four times. The final threshold was calculated as the mean of the last three threshold 
Simultaneous

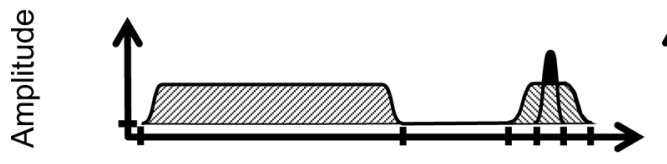

Precursor with 400-Hz notch (NNP4)
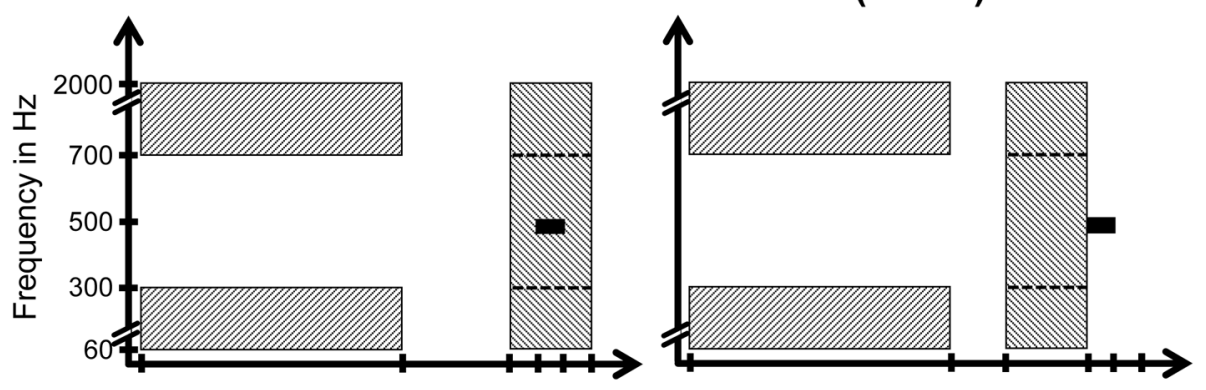

Precursor with $800-\mathrm{Hz}$ notch (NNP8)
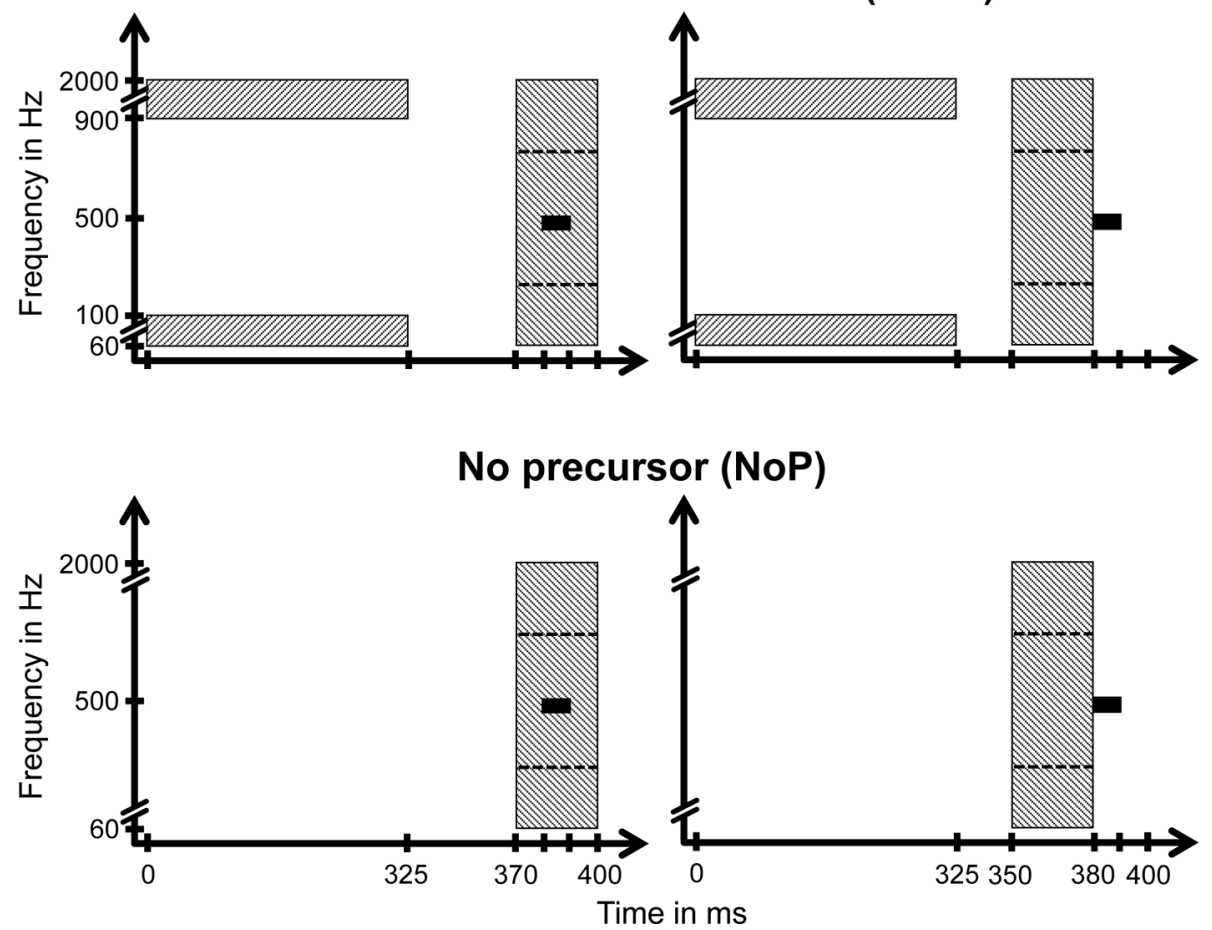

Figure 1. Schematic of temporal (1st row) and spectral characteristics (2nd-4th row) for simultaneous and forward-masking conditions, left and right columns, respectively. In both masking conditions, the temporal position of the precursor (from 0 to 325 ms; if present) and the 10-ms signal (black thick line in 2nd-4th row, onset $380 \mathrm{~ms}$ after interval onset) were fixed. The temporal position of the masker differed between the simultaneous-masking and the forward-masking conditions: for the forward-masking condition, the temporal position of the masker was shifted to an earlier point in time. The rectangle with left-sloping stripes surface in the $2 \mathrm{nd}-4$ th rows indicate the masker without a notch. The horizontal black dashed lines indicate the edges of the 400-Hz notch, which was also used as a masker notch width (apart from $0 \mathrm{~Hz}$ ). The precursor is indicated by rectangles with right-sloping stripes in the spectrograms of the 2nd and 3rd rows. The precursor conditions are abbreviated as NNP4 and NNP8, the condition with no precursor as NoP (4th row).

estimates. The first run of each condition was taken as a practice trial.

The listeners were seated in a double-walled soundproof booth. The experiment was controlled using MATLAB.
All stimuli were generated at a sampling frequency of $44.1 \mathrm{kHz}$. They were converted from digital to analogue signals and presented binaurally via an external sound card (RME Fireface 400, Haimhausen, Germany) and 


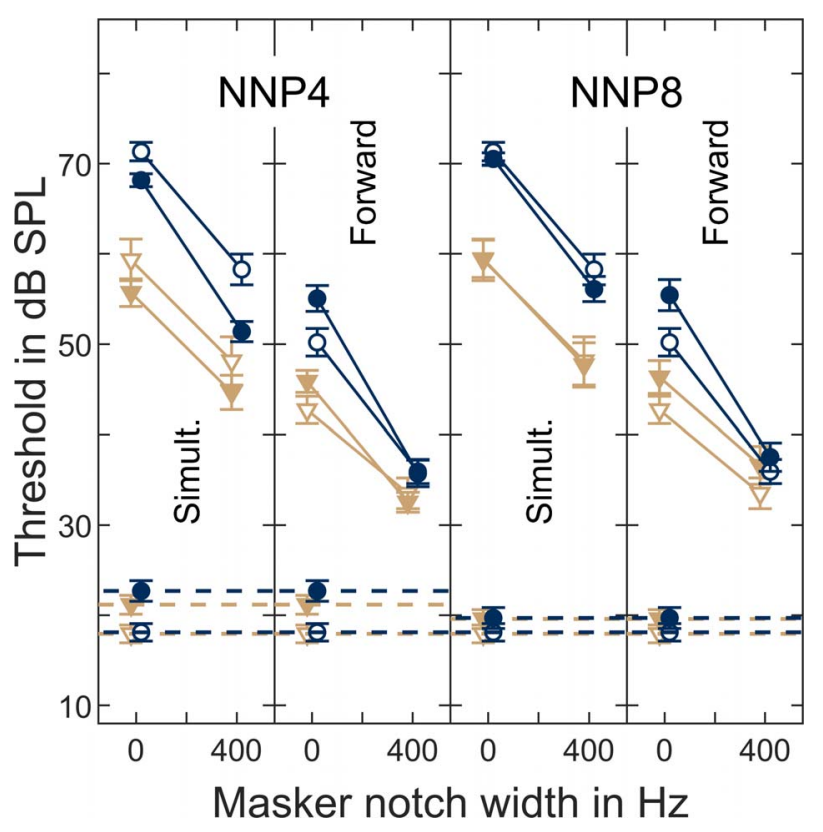

Figure 2. Mean data averaged across the individual data of thirteen listeners. The figure is subdivided in four panels. Each panel shows the results of a combination of precursor characteristic (NNP4 in first and second panel; NNP8 in third and fourth panel) and masking paradigm (simultaneous masking in first and third panel; forward masking in second and fourth panel). Open symbols indicate thresholds without a precursor (NoP), filled symbols those where the precursor was present (NNP). Thresholds for a diotic signal are marked with circles, those of the dichotic signal with downward-pointing triangles. In each panel, thresholds are shown as a function of masker notch width. Masked thresholds are indicated by data points connected with solid lines, whereas unmasked thresholds are shown with a symbols at the bottom left corner of the panels and horizontal dashed lines. Error bars indicate \pm 1 standard error. For a better visibility, diotic thresholds are slightly shifted to the right and dichotic thresholds to the left, respectively.

headphones (Sennheiser HDA200, Wedemark, Germany). The headphones were free-field equalized according to IEC $389-5$ (as in [3]).

\section{Results}

Figure 2 shows average thresholds as a function of the notch width for the NNP4 precursor (first two panels from the left) and the NNP8 precursor (third and fourth panel) in the simultaneous-masking (first and third panel) and forward-masking (second and fourth panel) conditions. The solid lines with the filled symbols indicate thresholds in the precursor conditions (NNP4 or NNP8). For comparison, open symbols show the corresponding no-precursor condition (NoP) (the same panel). The symbols in the left bottom of each panel and the dashed horizontal lines indicate thresholds obtained in the no masker condition.

Average thresholds in the absence of the masker increased by about $4 \mathrm{~dB}$ when the precursor with a $400 \mathrm{~Hz}$ notch was added, and by less than $2 \mathrm{~dB}$, when the precursor with the wider notch of $800 \mathrm{~Hz}$ was introduced. Without a precursor, diotic thresholds in the simultaneous-masking condition decreased by $13 \mathrm{~dB}$, as the notch width increased from 0 to $400 \mathrm{~Hz}$. For the corresponding simultaneous-masking dichotic condition, a decrease of $11 \mathrm{~dB}$ was observed. In the forward-masking conditions, the decrease was 14 and $9 \mathrm{~dB}$ for the diotic and dichotic condition, respectively. The effect of the precursor depended on the precursor condition, signal phase, and masking condition. Simultaneous-masked thresholds tended to decrease when the precursor with the smaller notch width (NNP4) was present (compared to the corresponding NoP condition), whereas simultaneous-masked thresholds for the NNP8 and NoP conditions were very similar. In the forward-masking condition, masked thresholds tended to increase when a precursor was present (NNP4 and NNP8). In general, the increase tended to be larger for the masker with no notch than for the masker with a $400-\mathrm{Hz}$ notch. In particular for the diotic conditions, the addition of the precursor tended to increase the effect of introducing a notch in the masker (simultaneous- and forward-masking conditions). For the dichotic conditions, this effect is only observed in the forward-masking data for NNP4. For the other dichotic conditions, the decrease in threshold as a notch is introduced in the masker is the same with and without a precursor.

The results were also analysed statistically and a summary of the key statistical results is shown in Table 1. To assess the contribution of any forward masking by the precursor (in the absence of the masker) on the detectability of the signal, the baseline conditions (relevant to both simultaneous- or forward-masking conditions) were first compared in a within-subject ANOVA. The baseline conditions were conditions in which thresholds for detectability of the $S_{0}$ or $S_{\pi}$ signal were measured alone (absolute threshold) or in the presence of a precursor with a $400-\mathrm{Hz}$ or $800-\mathrm{Hz}$ notch (symbols at the bottom left corner of the panels and horizontal dashed line). For the ANOVA, the two factors were signal phase (two levels: $S_{0}$ or $S_{\pi}$ ) and precursor condition (three levels: NoP, NNP4, and NNP8). There was a significant effect of signal phase $\left[F_{(1,12)}=19.23, p<0.01\right.$ with effect size, $\left.\left.\eta^{2}=0.62\right)\right]$, precursor $\left[F_{(2,24)}=30.76, p<0.001\right.$ with effect size, $\left.\eta^{2}=0.72\right]$ and a significant interaction between signal phase and precursor $\left[F_{(4,28)}=3.50\right.$, $p<0.05$ with effect size, $\eta^{2}=0.23$. Post hoc pairwise comparisons showed that the main effect of precursor did contribute to forward masking of the signal: Thresholds for detecting the signal $\left(\mathrm{S}_{0}\right.$ or $\left.\mathrm{S}_{\pi}\right)$ were significantly higher with addition of any precursor (NNP4 or NNP8) compared to thresholds for signal detection alone $(p<0.01)$. Thresholds for detecting the signal $\left(\mathrm{S}_{0}\right.$ or $\left.\mathrm{S}_{\pi}\right)$ were significantly higher for a precursor with a narrower notch (NNP4) compared to a wider notch of NNP8 $(p<0.01)$. Post-hoc pairwise comparisons to investigate the interaction between signal phase and precursor conditions showed that there was no significant difference between absolute thresholds for detectability of the $S_{0}$ and $S_{\pi}$ signal with NoP or NNP8. However, with a NNP4, thresholds for detection of the $\mathrm{S}_{0}$ signal were significantly higher than for detection of the $\mathrm{S}_{\pi}$ signal $(p<0.01)$. 
Table 1. Summary of the key statsistical tests. The first column displays the experimental conditions (baseline, simultaneous and forward masking), metrics obtained (BMLD and BMLD diff $)$ and the statistical tests (ANOVA and key pairwise comparisons). The second column shows the main factors, and the third column shows the level of significance. See main text for abbreviations.

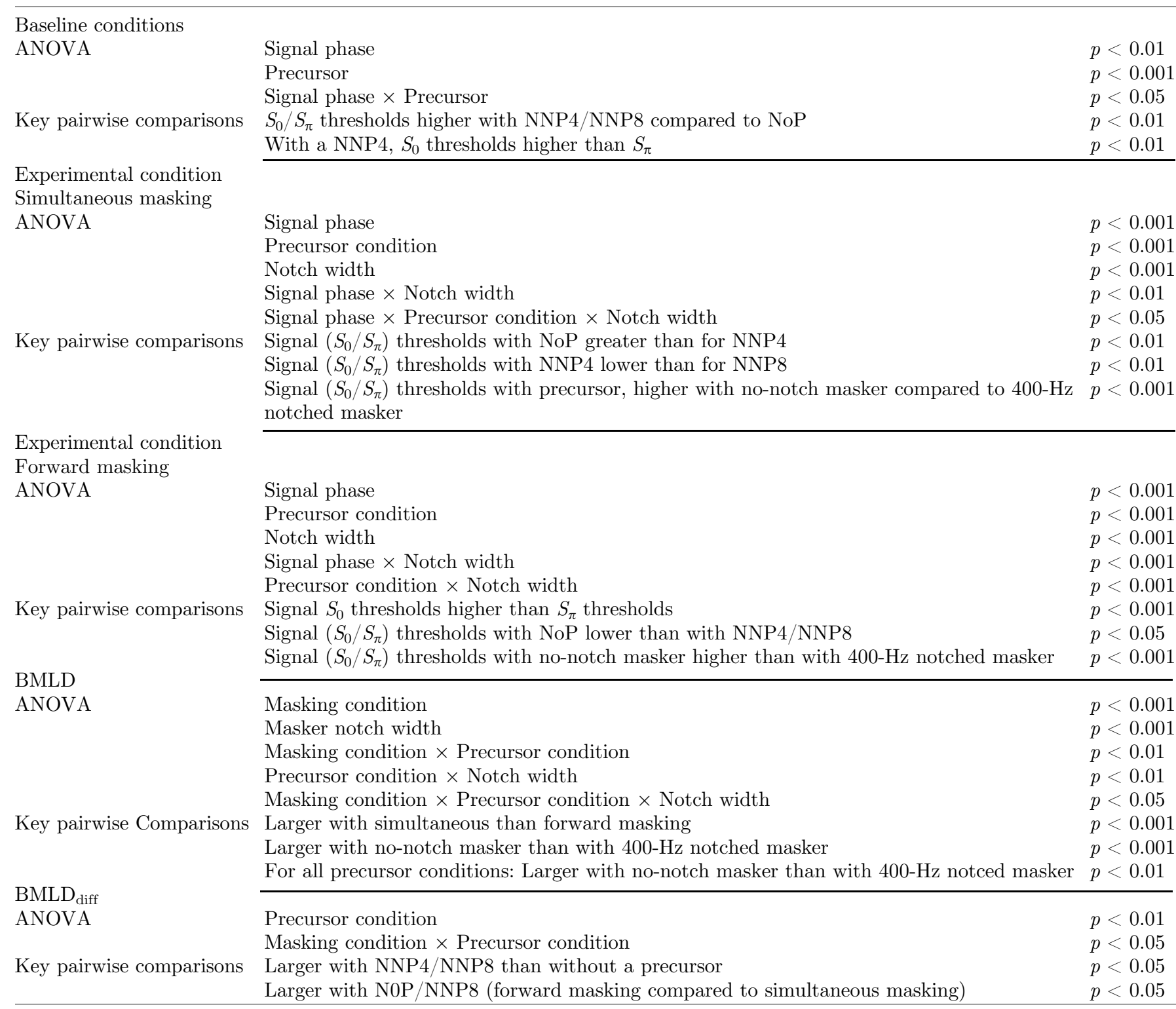

Within-subject ANOVAs were also conducted on the masked threshold values (forward and simultaneous). The experimental conditions were conditions in which detectability of the signal was measured in the presence of the simultaneous or forward masker (with no notch or a $400-\mathrm{Hz}$ notch) alone (NoP), or together with the precursor (NNP4 or NNP8). In the following data analyses of the simultaneous- and forward-masking data, Mauchly's Test of Sphericity was first initially conducted and shown not to be significant so sphericity could be assumed for the subsequent ANOVA analyses. Post-hoc pairwise comparisons were conducted with a Bonferroni correction to keep Type I error at $5 \%$.
For the ANOVA performed on the data from the simultaneous experiment, the three factors were signal phase (two levels: $S_{0}$ or $S_{\pi}$ ), precursor condition (three levels: NoP, NNP4, NNP8) and simultaneous masker notch width (two levels: masker with no notch, masker with a $400-\mathrm{Hz}$ notch). There was a significant effect of signal phase $\left[F_{(1,12)}=44.34\right.$, $p<0.001$ with effect size, $\left.\left.\eta^{2}=0.79\right)\right]$, precursor condition $\left[F_{(2,24)}=19.15, p<0.001\right.$ with effect size, $\left.\left.\eta^{2}=0.62\right)\right]$, simultaneous masker notch width $\left[F_{(1,12)}=282.81, p<0.001\right.$ with effect size, $\left.\eta^{2}=0.96\right)$ ], a significant two-way interaction between signal phase and simultaneous masker notch width $\left[F_{(1,12)}=20.49, p<0.01\right.$ with effect size, $\left.\eta^{2}=0.63\right]$ and a significant three-way interaction between signal phase, 
precursor condition and simultaneous masker notch width $\left[F_{(2,24)}=5.46, p<0.05\right.$ with effect size, $\left.\eta^{2}=0.31\right]$.

Post hoc pairwise comparisons on the main effect of the signal phase showed that simultaneous $S_{0}$ thresholds (across simultaneous masker and precursor conditions) were significantly higher than for the $\mathrm{S}_{\pi}$ signal $(p<0.001)$. For the main effect of precursor condition, simultaneous-masked thresholds for detecting the signal $\left(\mathrm{S}_{0}\right.$ or $\left.\mathrm{S}_{\pi}\right)$ in the NoP condition were significantly higher than simultaneousmasked thresholds in the NNP4 condition $(p<0.01)$ and simultaneous-masked thresholds in the NNP4 condition were significantly lower than in the NNP8 condition $(p<0.01)$. For the main effect of masker notch width, thresholds for detection of the signal $\left(\mathrm{S}_{0}\right.$ or $\mathrm{S}_{\pi}$ across precursor conditions) obtained with a simultaneous masker without a notch were higher than simultaneous-masked thresholds obtained when the masker had a $400-\mathrm{Hz}$ notch $(p<0.001)$.

Post hoc pairwise comparisons to investigate the threeway interaction showed that addition of a precursor with a 400-Hz notch (NNP4) significantly lowered thresholds for detecting an $S_{0}$ signal in the presence of a simultaneous masker (without or with a $400-\mathrm{Hz}$ notch) compared to in the absence of a precursor (NoP condition, $p<0.05$ ). Furthermore, the reduction in thresholds for the $\mathrm{S}_{0}$ signal in the presence of a simultaneous masker (with a $400-\mathrm{Hz}$ notch), was significantly greater with the addition of a NNP4 rather than an NNP8 $(p<0.05)$. Similarly, when detecting an $\mathrm{S}_{\pi}$ signal in the presence of a simultaneous masker (without a notch) the reduction in thresholds was significantly greater with the addition of a NNP4 compared to NoP $(p<0.01)$ or addition of a NNP8 $(p<0.05)$.

The forward-masking data were analysed in the same way as the simultaneous-masking data. The threshold values obtained in the baseline conditions were not statistically (re-)analysed since they are the same as for the simultaneous-masking experiment.

A within-subject ANOVA was conducted on the threshold values for the conditions in which detectability of the signal was measured in the presence of the forward masker (with no notch or a 400-Hz notch) for all precursor conditions (NoP, NNP4, NNP8). For the ANOVA, the three factors were: signal phase (two levels: $\mathrm{S}_{0}$ or $\mathrm{S}_{\pi}$ ), precursor condition (three levels: NoP, NNP4, NNP8) and forwardmasker notch width (two levels: masker with no notch, masker with a $400-\mathrm{Hz}$ notch). There was a significant effect of signal phase $\left[F_{(1,12)}=30.81, p<0.001\right.$ with effect size, $\left.\left.\eta^{2}=0.72\right)\right]$, precursor condition $\left[F_{(2,24)}=15.78, p<0.001\right.$ with effect size, $\left.\eta^{2}=0.57\right)$ ], forward-masker notch width $\left[F_{(1,12)}=454.18, p<0.001\right.$ with effect size, $\left.\left.\eta^{2}=0.97\right)\right]$, and significant two-way interactions between signal phase and forward-masker notch width $\left[F_{(1,12)}=42.05\right.$, $p<0.001$ with effect size, $\left.\eta^{2}=0.78\right]$ as well as precursor condition and forward-masker notch width $\left[F_{(2,24)}=13.01\right.$, $p<0.001$ with effect size, $\left.\eta^{2}=0.52\right]$.

Post hoc pairwise comparisons on the main effect of signal phase showed that $\mathrm{S}_{0}$ thresholds (across forward-masker notch widths and precursor conditions) were significantly

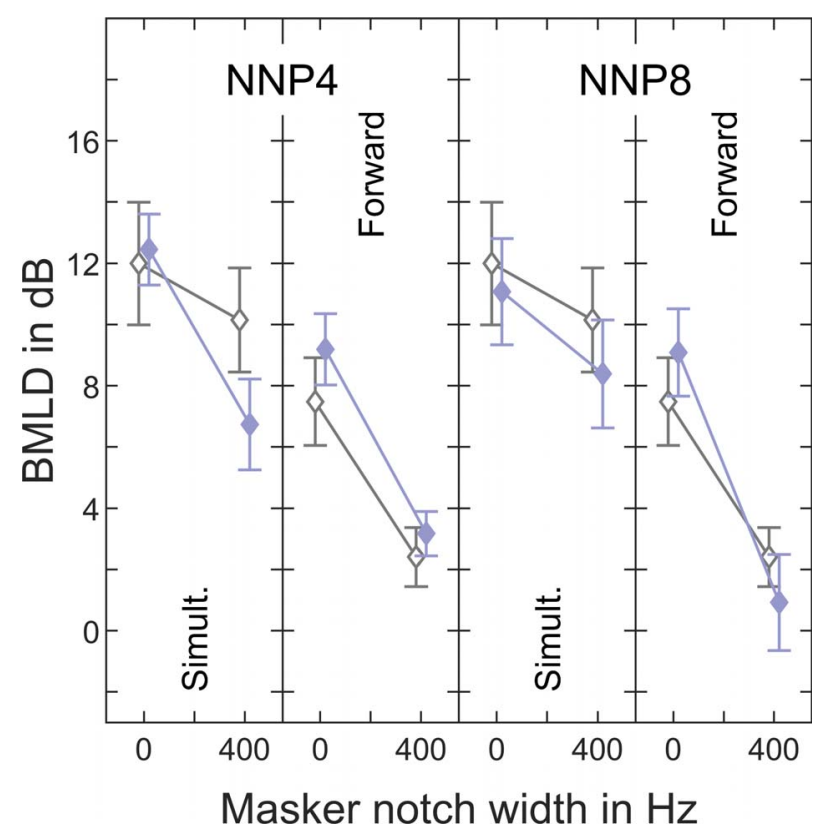

Figure 3. Mean binaural masking level differences (BMLDs) averaged across the individual BMLDs of the thirteen listeners. Left two columns show data for the NNP4 condition, right two columns for the NNP8 condition. Open symbols indicate the BMLD without a precursor, filled symbols indicate conditions where the precursor (with a notch width of $400 \mathrm{~Hz}$ and $800 \mathrm{~Hz}$, respectively), was present. To aid visibility, the BMLDs without a precursor (open symbols) are slightly shifted to the left and those with a precursor (filled symbols) are shifted to the right. Error bars indicate plus and minus one standard error.

higher than $\mathrm{S}_{\pi}$ thresholds $(p<0.001)$. For the main effect of precursor condition, thresholds for detecting the signal $\left(\mathrm{S}_{0}\right.$ or $\mathrm{S}_{\pi}$ across forward-masking conditions) in the NoP condition were significantly lower than thresholds in the NNP4 $(p<0.05)$ and NNP8 condition $(p<0.001)$.

For the main effect of forward-masker notch widths, thresholds for detection of the signal $\left(\mathrm{S}_{0}\right.$ or $\mathrm{S}_{\pi}$ across precursor conditions) obtained with a forward masker without a notch were significantly higher than thresholds obtained when the forward masker had a 400-Hz notch, NNP4 $(p<0.001)$.

Post hoc pairwise comparisons to investigate the twoway interaction between precursor condition and forwardmasker notch width showed that thresholds for detection of the signal $\left(\mathrm{S}_{0}\right.$ or $\left.\mathrm{S}_{\pi}\right)$ were significantly higher in the presence of a forward masker without a notch than with a $400-\mathrm{Hz}$ notch, irrespective of the absence or presence of a precursor (with either notch) $(p<0.001)$.

Figure 3 shows the average BMLD. The organization of the panels is the same as in Figure 2, i.e., the first and third panel show simultaneous-masked thresholds, the second and fourth panel thresholds in the forward-masking condition. Open symbols indicate thresholds in the NoP condition, filled symbols those in the conditions with precursor (NNP4 in the first two panels, NNP8 in the third and fourth panel). 
The BMLD tended to slightly decrease in the presence of a precursor in the simultaneous-masking condition and tended to increase in the forward-masking condition. The effects were usually small $(\leq 2 \mathrm{~dB})$, except with the 400 $\mathrm{Hz}$ masker notch width in the first panel, where the BMLD decreased by about $3 \mathrm{~dB}$ when the precursor was added (NNP4 vs. NoP). In general, the precursor tended to increase the effect of introducing a notch in the masker on the BMLD.

A within-subject ANOVA was conducted on the BMLD values to compare the extent of the BMLD across simultaneous- and forward-masking conditions, as well as precursor and masker notch widths. For the ANOVA, the three factors were: masking condition (two levels: forward masking or simultaneous masking), precursor condition (three levels: NoP, NNP4, NNP8) and masker notch width (two levels: masker with no notch, masker with a $400-\mathrm{Hz}$ notch).

There was a significant effect of masking condition $\left[F_{(1,12)}=22.35, p<0.001\right.$ with effect size, $\left.\left.\eta^{2}=0.65\right)\right]$, masker notch width $\left[F_{(1,12)}=80.46, p<0.001\right.$ with effect size, $\left.\left.\eta^{2}=0.87\right)\right]$, and significant two-way interactions between masking condition and precursor condition $\left[F_{(2,24)}=6.21, p<0.01\right.$ (two-tailed) with effect size, $\left.\eta^{2}=0.34\right]$ as well as precursor condition and masker notch width $\left[F_{(2,24)}=6.14, p<0.01\right.$ with effect size, $\left.\eta^{2}=0.34\right]$. There was also a three-way interaction between masking condition, precursor condition and masker notch width $\left[F_{(2,24)}=3.47, p<0.05\right.$ with effect size, $\left.\eta^{2}=0.224\right]$.

Post hoc pairwise comparisons on the main effect of masking condition showed that (across precursor condition and masker notch widths) BMLDs derived using simultaneous masking were significantly larger than the BMLD derived using forward-masked thresholds $(p<0.001)$.

Post hoc pairwise comparisons on the main effect of masker notch width, showed that BMLDs (across masking and precursor conditions) derived with a no-notch masker were significantly larger than the BMLDs derived using a masker with an 400-Hz notch $(p<0.001)$.

Post hoc pairwise comparisons to investigate the twoway interaction between masking condition and precursor condition showed that (across masker notch-width conditions) for each precursor condition (NoP, NNP4, NNP8) BMLDs derived using simultaneous masking were larger than BMLDs derived using forward masking $(p<0.01)$.

Post hoc pairwise comparisons to investigate the twoway interaction between masker notch width and precursor condition showed that (across simultaneous- and forwardmasking conditions) in all precursor conditions (NoP, NNP4, NNP8), BMLDs were larger when the masker had no notch than when the masker had a 400-Hz notch $(p<0.001)$.

Post hoc pairwise comparisons to investigate the threeway interaction between masking conditions, masker notchwidth conditions and precursor conditions showed that in the absence of a precursor (NoP), or presence of a precursor (NNP4 and NNP8), when presented with a masker with a 400-Hz notch, BMLDs were always significantly larger when derived using simultaneous masking than when using forward masking $(p<0.05)$. The exception to this trend occurred only for the case when an $800-\mathrm{Hz}$ precursor was presented together with a masker without a notch, in which case there was no significant difference in the BMLDs derived using simultaneous and forward masking.

The decrease in BMLD between the no-notch masker and the $400-\mathrm{Hz}$ notch masker indicates that the effective filter of the monaural system is sharper than the effective filter of the binaural system. Addition of the precursor results in an increase in the effect of introducing a notch in the masker on the BMLD, indicating a change of the relation between the effective monaural and binaural filter shape.

To obtain estimates of the change in relation between the effective filter shapes of the monaural and binaural system, the BMLD values obtained in the presence of a masker with a $400-\mathrm{Hz}$ notch were subtracted from the BMLD values obtained in the presence of a masker without a notch (per masking conditions of forward or simultaneous masking). This difference will be referred to as BMLD diff.

For the ANOVA, the two factors were masking condition (two levels: forward or simultaneous) and precursor (three levels: NoP, NNP4, NNP8). There was a significant effect of precursor $\left[F_{(2,24)}=6.14, p<0.01\right.$ with effect size, $\left.\left.\eta^{2}=0.34\right)\right]$ and a significant interaction between masking condition and precursor $\left[F_{(2,24)}=3.47, p<0.05\right.$ with effect size, $\eta^{2}=0.22$. Post hoc pairwise comparisons showed that (across masking conditions; forward and simultaneous masking) the $\mathrm{BMLD}_{\text {diff, values were significantly larger }}$ with the addition of a precursor (NNP4 or NNP8) $(p<0.05)$. Thus, the difference in effective filter shape between the monaural and binaural system increases in the presence of the precursor. Post hoc pairwise comparisons to investigate the two-way interaction between masking conditions and precursor conditions showed that the $\mathrm{BMLD}_{\text {diff }}$ values were significantly larger with $\mathrm{NoP}$ or NNP8 when using forward masking compared to simultaneous masking ( $p<0.05)$, with a mean difference (forward masking minus simultaneous masking) of $3.23 \mathrm{~dB}$ (with $\mathrm{NoP}$ ) and $5.48 \mathrm{~dB}$ (with a NNP8). Thus, in the NoP and NNP8 conditions (but not in the NNP4 condition), the difference between the filter shape of the monaural and binaural system was more pronounced when using forward masking compared to simultaneous masking.

\section{Discussion}

The present study investigated the effect of a precursor on binaural notched noise data. In the following subsections, different aspects of the data are discussed. The first three subsections focus on the comparison to previous studies with respect to spectral masking without a precursor (Sect. 4.1), the masking due to the precursor without an additional masker (Sect. 4.2), and spectral masking with a precursor (Sect. 4.3). The last two subsections discuss the data in the light of different hypotheses of the underlying mechanisms, in general (Sect. 4.4), and in the specific light of the MOC reflex (Sect. 4.5). 


\subsection{Comparison to previous spectral masking data without precursor}

For all masking conditions (simultaneous or forward), changing the masker from a band limited masker without a notch, to one with a $400-\mathrm{Hz}$ notch at the signal frequency, significantly reduced masked thresholds, which is consistent with previously published literature data (e.g., [35]) and reflects auditory frequency selectivity.

In the present study, for the simultaneous-masking condition, diotic thresholds decreased by about $13 \mathrm{~dB}$ when a masker notch of $400 \mathrm{~Hz}$ was introduced, compared to the masking condition without a notch. Commonly, a decrease of $20 \mathrm{~dB}$ is measured for this change in notch width with a signal frequency of $500 \mathrm{~Hz}$ (e.g., [39, 40]). This reduced effect of notch width on threshold is presumably a consequence of the signal duration of $10 \mathrm{~ms}$ that is considerably shorter than the several hundred milliseconds commonly used in notched-noise experiments. A comparable decrease in threshold was found in Verhey et al. [3] for their 25-ms long signal when taking into account the higher signal frequency in their study; at the same relative change in notch width, threshold decreased by about the same amount as in the present study.

Introducing an interaural signal phase difference of $\pi$ resulted in a significant threshold reduction of $12 \mathrm{~dB}$ for the no-notch condition. Thus, as intended, the BMLD is considerably larger than in [3]. This is mainly due to the use of a $500-\mathrm{Hz}$ signal in the present study compared to $1 \mathrm{kHz}$ in [3]. In addition, the larger BMLD in the present study than in [3] may be partly due to the gating of signal and masker. Signal and masker both had a duration of $25 \mathrm{~ms}$ and were gated on and off simultaneously with 12.5-ms long raised cosine ramps in [3]. In contrast, a fringe condition with asynchronous gating of signal and masker was used in the present study. As shown by Robinson and Trahiotis [41], simultaneous gating of signal and masker reduces the BMLD compared to a fringe condition (as was used in the present study).

For all conditions of the present study, the BMLD for the $400-\mathrm{Hz}$ notched-noise masker was smaller than the BMLD for the no-notch masker. Thus, the effect of introducing a notch in the masker was stronger for the diotic condition than for the corresponding dichotic condition. This finding is in agreement with previous spectral masking experiments using a simultaneous notched-noise masking paradigm [3, 38-40, 42]. The decrease in threshold as a notch is introduced is smaller for the dichotic condition than for the diotic condition, indicating that masker energy outside the monaural auditory filter affects dichotic thresholds. An explanation is that a retro-cochlear across-frequency process yields to a wider effective binaural bandwidth (e.g., [36]). The exact mechanism is still unclear. For example, Nitschmann and Verhey [40] showed two possible realizations of an across-frequency process that predicted the effective wider binaural bandwidth. The present study extends the previous data sets on binaural notched noise experiments to conditions where signal and masker are not presented simultaneously (forward-masking condition). As for the simultaneous-masking condition, dichotic thresholds are lower than the corresponding diotic thresholds. The difference between the two thresholds, i.e., the BMLD, is about $8 \mathrm{~dB}$ for the no-notch condition, i.e. significantly smaller than in the corresponding simultaneous-masking condition. The finding of a positive BMLD when signal and masker are not presented simultaneously is in agreement with previous forward-masking data where broadband uniform-exciting noise maskers were used [43, 44]. This indicates that binaural cues are still used for signal detection and that the binaural benefit does not result from simultaneous interaction of masker and signal within the auditory filter.

For the NoP condition, the decrease in threshold as a notch is introduced in the masker is larger for the diotic forward-masking condition than for the corresponding simultaneous condition indicating higher frequency selectivity in the forward-masking condition. Similar results were observed by $[45,46]$. Experiments conducted to derive psychoacoustical tuning curves with a signal presented after masker offset also indicate a higher frequency selectivity. This difference in frequency selectivity is presumably at least partly due to two-tone suppression, which reduces the signal in the simultaneous condition, but does not affect the signal in the forward-masking condition [47, 48]. The BMLD was overall significantly larger in the simultaneous masking condition than the forward-masking condition (12 dB vs. $8 \mathrm{~dB}$ for the no-notch masker). For a 20-ms signal, an even larger reduction was found in [49] (17 dB vs. $9 \mathrm{~dB})$. Differences between the results of the present study and [49] are maybe due to individual differences and due to the shorter signal duration of the present study. For example, Yama [50] measured individual BMLDs of 3-6 dB (average $4 \mathrm{~dB}$ ) for a $15-\mathrm{ms} 500 \mathrm{~Hz}$ tone pip. This data set also show the large interindividual differences for BMLDs with short durations. Despite a training phase of $20 \mathrm{~h}$, the BMLDs for $250 \mathrm{~Hz}$ ranged from $-2 \mathrm{~dB}$ to about $8 \mathrm{~dB}$.

The smaller BMLD in the forward-masking condition than in the simultaneous-masking condition for the masker with no notch may be due to the effect of the masker level on the BMLD. The BMLD for a fixed-spectrum masker decreases with masker spectrum level towards lower masker spectrum levels (see, e.g. [42]). However, this level effect cannot account for the change in BMLD as the notch is introduced into the masker. This was shown by Nitschmann et al. [42] by comparing the data of the masker-level experiment and the notched-noise experiment at the same diotic threshold. This comparison was motivated by the power spectrum model [33] that assumed that the portion of the masker falling within the passband of the auditory filter centered at the signal frequency determines diotic threshold. Nitschmann et al. [42] showed that for a masker with a notch, the BMLD was smaller than expected from the masker-level-dependent BMLD.

\subsection{Masking due to the precursor}

Verhey et al. [3] discussed the possible role of the precursor as a masking sound. They showed that the preceding 
precursor masked the diotic and dichotic signals on average by $9 \mathrm{~dB}$ in absence of the simultaneous masker. Although this was at least $15 \mathrm{~dB}$ below the thresholds in the presence of the simultaneous masker, the precursor may still have had a substantial effect on the signal representation in the absence of the masker. Thus, one aim of the present study was to reduce the masking effect due to the presence of the precursor. Verhey et al. [3] used a band-limited precursor (60-2000 Hz) with a level of $63 \mathrm{~dB}$ SPL, which was presented $50 \mathrm{~ms}$ before signal onset. The silent interval between precursor offset and signal onset was about the same in the present study $(55 \mathrm{~ms})$. The precursor level was set to $61-62 \mathrm{~dB}$, depending on the precursor notch, i.e., was also comparable. Nevertheless, the masking effect of the signal due to the precursor was considerably less than the $9 \mathrm{~dB}$ observed in Verhey et al. [3], as intended. This was achieved by the introduction of a spectral gap $(400 \mathrm{~Hz}$ and $800 \mathrm{~Hz}$ ) in the noise, which was arithmetically centred at the signal frequency. Thereby, the increase of thresholds without a masker due to the presence of the precursor was significant, but only $3-4 \mathrm{~dB}$ for the $400-\mathrm{Hz}$ notch and even lower (1-2 dB) for the $800-\mathrm{Hz}$ notch. Thus, although not completely abolished, forward masking of the precursor was considerably reduced in the present study and should thus not have affected the thresholds where the masker was present, at least not due to energetic masking.

\subsection{Comparison to previous notched-noise data with a precursor}

Zhou et al. [51] measured the effect of a 1000-ms precursor (notched- or bandpass noise) on gain modulation and measured distortion-product OAEs (DPOAEs). They found that measures of sensitization (difference between perceptual thresholds with bandpass and notched-noise precursors) correlated with DPOAEs measured with the lowest level primary tone (25 dB SPL). They also showed that a notched-noise precursor improves detection thresholds (as measured psychophysically) compared to the no-precursor condition (see also [2]). Zhou et al. [51] suggested that these sensitization effects could be mediated at the level of the $\mathrm{OHC}$, but also acknowledged that since the precursor effect lasted as late as $250 \mathrm{~ms}$, that the effects were likely mediated by the MOC. We show a similar reduction in simultaneous-masked thresholds for the signal with notched noise precursor (NNP4) compared to the no precursor (NoP) condition.

The effect of the precursor tended to be larger for the diotic condition than for dichotic condition. This is in agreement with Verhey et al. [3], which had a similar aim as the present study. However, with respect to the combined effect of precursor and notch width, the results of the present study appear to contradict our previous study [3]. Verhey et al.'s [3] threshold curves were shallower when the precursor was present whereas the present data show the opposite effect, i.e., a steeper slope of the line connecting thresholds when the precursor is present. A major difference between the two studies is the spectrum of the precursor. Whilst the precursor in the present study had a notch, there was no notch in the precursor used by Verhey et al. [3]. Indeed, several previous studies using a notchednoise precursor agree with the current findings. For example, Carlyon [52] described an experiment similar to the diotic simultaneous-masking condition of the present study, where thresholds for a short $1-\mathrm{kHz}$ tone pip centred in a simultaneously-gated masker with, or without, a preceding signal, i.e., a precursor (referred to as a "primer" in Carlyon's study) were measured. In one condition, the precursor had the same spectrum as the masker. For the no-notch condition, thresholds were almost the same in the precursor and no-precursor condition. The decrease in threshold, as the notch width increased, was faster in the precursor condition than in the no-precursor condition. This was later confirmed by Hicks and Bacon [53] for signal frequencies of $1 \mathrm{kHz}$ and $4 \mathrm{kHz}$. A conceptual difference between the experimental paradigms of these two studies and the present study relates to the precursor spectrum that was adjusted to match the masker spectrum, whereas in the present study the same precursor spectrum was used for the two masker notch widths. Nevertheless, the results for the diotic simultaneous condition are remarkably similar to those of the previous study, especially when the precursor had a $400-\mathrm{Hz}$ notch (NNP4). In this condition, the precursor and the masker with a $400-\mathrm{Hz}$ notch had the same spectrum but the spectra differed for the no-notch masker. Carlyon [52] and Hicks and Bacon [53] showed that a difference in spectral characteristics between a precursor and masker tends to decrease the threshold-reducing effect of the precursor. Since there was no effect of precursor for the no-notch masker with the spectrum-matched precursor, one may not expect a precursor effect in the present study for the no-notch masker. In contrast, the present data indicate a significant reduction in diotic simultaneous-masked thresholds for both notch widths. One may, however, explain the reduced precursor effect in the NNP8 condition with the larger spectral difference between precursor and masker.

All of the above mentioned literature experiments referred to simultaneous-masking conditions. In contrast, Jennings and Strickland [31] investigated the effect of a precursor on notched-noise masking where the signal was presented after masker offset, i.e. in a forward-masking condition. The masker level was varied to determine threshold. A lower masker level was required to mask the signal in the precursor condition. This was presumably due to the choice of the precursor. The precursor was a pure tone with the same frequency as the signal. It had already been shown by Carlyon [52] that a precursor with a similar spectrum as the signal has a detrimental effect on signal detection. Interestingly, Jennings and Strickland [31] measured a shallower threshold curve for the precursor condition than for the non-precursor condition. Thus, the data seem to indicate a reduced spectral selectivity in the presence of the precursor. Similar results were also shown by Verhey et al. [3] for a bandpass-noise precursor. In contrast, all data sets with a notched-noise precursor, including the results of the present study, seem to indicate higher frequency selectivity. Thus, the spectrum of the precursor seems to determine the direction of the precursor effect. 


\subsection{Precursor effect in the light of various proposed underlying processes}

Earlier studies have usually investigated the effects of a precursor/inducer effect in monaural experiments, using a variety of methods (psychoacoustcial/OAE), stimuli (tone or multitone precursor/masker) and masking paradigms (forward or simultaneous masking). The conclusion differs between the studies, ranging from, neural adaptation [54], moderate enhancement effects [55], small enhancement effects [56], to gain reduction due to the activation of the MOC reflex by the precursor [57].

The enhancement effect is distinct from the effect measured in the current study. The enhancement effect (e.g., [58]) refers to the increased audibility of a given target signal component of a spectral complex by prior exposure to the spectral complex with the target signal component deleted; this is suggested to be due to an increase in gain in the (neutrally) unadapted frequency region (adaptation inhibition). The latter example refers to enhancement in the case of a single unmasked target tone. Other studies (e.g., [59]) have measured the enhancement effect for a target tone presented in a simultaneous masker, where the simultaneous masker and the precursor (inducer) complexes have an equivalent spectral notch width centred on the target signal. In the current study, the target tone is presented with a simultaneous or forward masker; these noise maskers do not have a notch centred on the target signal. The precursor in the current study also did not have a spectral notch centred on the target signal and is analogous in design to the enhancer complexes used in previous studies (e.g., [60]). However, in the current study the precursor notch was much wider than just the frequency range encompassing the target signal frequency. The precursor notch width extended either side of the target frequency; extending over much of the frequency range of the simultaneous and forward masker components. The efferent effect (elicted by the precursor) is suggested to operate across a relatively wide frequency range. It is for that reason that a notch was introduced into the precursor, to minimise forward masking due to the precursor, yet allow for gain reduction due to the precursor acting to activate the efferent response. In the current case if the precursor had acted as an enhancer (by way of adaptation inhibition) then gain would be increased for both the target signal and the simultaneous masker components around the target signal frequency and there would be expected to be no change in masked signal threshold. In the case of the forward masking condition, this would mean that the gain would be increased for the forward masker components around the target signal frequency and detectability of the target signal would be reduced. Target signal detectability would be further reduced as the precursor notch was widened. The results show effects that differ from purely an enhancer effect.

Direct comparisons between our precursor study using diotic and dichotic stimuli and previous precursor/ enhancer/elicitor studies (using psychophysics or OAE) are problematic due to the range of stimuli (often monotic) and methods used in previous studies. Thus, in the following discussion, only a few studies are discussed with respect to the present results. Almishaal et al. [61] showed that a notched-noise precursor improves amplitude-modulation (AM) detection of a subsequently presented narrow-bandnoise carrier centred at $5 \mathrm{kHz}$, which they posited was due to a reduction in gain due to the MOC reflex. Almishaal et al. [61] also acknowledged that some of their results could be possibly accounted for, if the notched-noise used to restrict off-frequency listening, had itself contributed to facilitating an enhancement effect, and/or dynamic range adaptation had been activated at the higher levels of the auditory system. Marrufo-Pérez et al. [62] used words in noise as stimuli and suggested that the word enhancement could be explained by a neural adaptation process, distinct from an activation of the MOC reflex. Using three types of precursors (two-octave band noise, inharmonic complex tone, and pure-tone), Wojtczak et al. [63] investigated the role of the MOC reflex on AM detection for a 1- and $6-\mathrm{kHz}$ carriers at three levels, in two-octave wide noise. Overall, the noise precursor produced a large improvement in AM detection at all three levels tested. Comparison of these psychophysical results with stimulus frequency OAEs using the same stimuli, appeared to suggest that observed precursor effects may not have been solely due to efferent mediated gain reduction, but rather may be mediated by higher-levels of the auditory system.

Carlyon [52] interpreted their data in the light of highlevel grouping processes, where groups are formed in frequency regions containing common amplitude envelopes, enhancing the signal representation of newly-arriving energy in previously unstimulated frequency regions. According to this hypothesis, the effect of the precursor should be larger for an equal spectrum of precursor and masker (i.e., masker and precursor have a $400-\mathrm{Hz}$ notch), since then only the signal changes the spectrum. However, the simultaneous-masked thresholds of the present study decrease for both masker notch widths, i.e., in contrast to the hypothesis also for the no-notch masker.

Moreover, on the basis of a central grouping process, one may assume that grouping of masker and precursor with the same notch width should be stronger in the forward-masking condition (due to the smaller temporal gap between these two stimuli) than in the simultaneous-masking condition. Thus, the subsequent signal should be even easier to detect (improved "pop-out" due to signal delay) than in the simultaneous-masking condition, in which the precursor-masker grouping and signal "pop-out" happens at the same time. However, the opposite trend is observed in the data.

The grouping hypothesis can also not explain a larger effect of the precursor on the diotic than on the dichotic simultaneous-masking condition. However, one may reconcile the data with this hypothesis by assuming that the binaural cues are so strong that the signal and masker are already perceptually separated and thus an additional grouping cue cannot enhance the signal representation further. In a similar vein, one may argue that the negligible effect of the precursor on the masking data with the $400-\mathrm{Hz}$ notch when the signal was presented after masker offset 
(forward-masking condition) can be explained on the basis of a grouping cue, since this asynchronous stimulus presentation prevents grouping from signal and masker. Note, however, that even in the simultaneous condition signal and masker were not gated on and off simultaneously, which should reduce, if not abolish, a grouping due to common onset. In addition, the grouping hypothesis cannot account for the increase in threshold for the no-notch masker in the diotic forward-masking condition when the precursor was added. Carcagno et al. [64] investigated the possible role of grouping-like cues for the signal detectability in a multitone masker preceded by a precursor (which either had similar or dissimilar spectral components to the masker) and found that higher-level grouping cues may be insufficient in accounting for all conditions of signal enhancement due to precursor presentation. This supports the hypothesis that lower level processes such as the MOC reflex are involved in such precursor masking paradigms.

\subsection{The results in the light of the MOC reflex}

A broadening of the frequency selectivity due to the activation of the MOC reflex by the precursor [3] is consistent with the finding that this efferent activation of the cochlea linearises cochlear processing. The opposite effect, i.e., a steepening of the threshold curve as observed in the present study, seems to be add odds with this interpretation. However, the present data can still be reconciled with a change in cochlear processing due to the MOC reflex, if a frequency specificity of this reflex is taken into account. Such frequency specificity was reported in [65] using SFOAEs and [66] using a pyschophyical methodology. The gain reduction (and thus the change in cochlear processing) is largest if the precursor frequency is close to the signal frequency. As the spectral distance between precursor frequency and signal frequency increases, the effect of the precursor on cochlear gain decreases. Figure 4 shows how this additional property of the MOC reflex may account for the effect of precursor in the different diotic conditions of the present study. The signal (pointed arrow) is only shown for the simultaneous-masking condition. The top rows show the spectrum for the no-notch masker (left) and the masker with a $400 \mathrm{~Hz}$ notch (right). The middle rows show the corresponding cochlea excitation without precursor. Due to cochlear gain, masker and signal are amplified (gain is represented by the thick black filled arrows). On the other hand (two-tone) suppression reduces the middle portion of the no-notch masker (left) and signal (suppression is represented by the thick open arrows). This does not change the signal-to-noise ratio since both signal and the masker portion that is most relevant for the masking are suppressed. For the simultaneous masker with a $400-\mathrm{Hz}$ notch, only the signal is suppressed, hence a higher threshold than without suppression. Signals in a forwardmasking condition are not suppressed for both masker notch widths, i.e., the threshold curve is steeper than in diotic simultaneous condition.

In the bottom rows, the change in excitation due to the addition of the precursor is shown. The precursor activates the MOC reflex, which reduces cochlear gain. Since the reduction has a certain frequency specificity [66] it mostly affects the portion at the edges of the masker. This also reduces suppressive effect within the no-notch masker, leading to higher masking of nonsimultaneously presented signal. For $400-\mathrm{Hz}$ notched-noise masker, the signal in the simultaneous condition is better audible due to reduced gain and suppression. Strickland [13] also showed, using a monaural signal (of short and long duration) and simultaneous notched-noise masker with a backward fringe or backward plus forward fringe, a decrease in gain at the signal frequency, with a possible decrease in suppression of the signal. The reduced effect of the precursor with the $800-\mathrm{Hz}$ notch compared to that with a $400-\mathrm{Hz}$ notch can also be understood when a frequency specific gain reduction due to the precursor is assumed: The spectral edges of the notch are further away and hence the gain reduction is not as severe as it is for the precursor with the $400-\mathrm{Hz}$ notch. Thus, the steepening of the threshold curve is presumably not just an indication of a sharper effective filter but rather a combination of frequency selectivity of the MOC reflex and suppression.

The above explanation can not directly account for the difference in the effect of precursor on diotic and the dichotic thresholds. Diotic thresholds seem to be more affected by the precursor than the dichotic thresholds resulting in significant steepening of the slope of the line connecting the BMLDs. A slightly larger effect on the diotic than on the dichotic thresholds is in agreement with [3], although in that study the precursor made estimation of the effective filter width more similar for the diotic and dichotic data. In the light of an explanation based on the MOC reflex, this may be interpreted as evidence for a specific binaural input to the MOC reflex. One suggestion is that a role of the efferent system is to maintain binaural cues useful for signal detection and in sound localisation [66]. The present data are in agreement with [3] in the sense that the binaural frequency selectivity seems to be affected differently from the monaural system when the MOC reflex is activated.

Overall, an understanding of how binaural hearing is affected by the MOC reflex will be of value to the development of models of human auditory binaural perception as well as contributing to recent models of loudness coding (e.g., $[68,69])$. Current binaural models [30] are unable to fully account for the effects of the MOC reflex on binaural cue processing. Efferent effects are likely to contribute to binaural processing in complex ways [21]. For instance, models that rely on averaging temporal information over a temporal window longer than the brief signal [24] are not able to fully account for efferent-mediated gain reduction at the auditory periphery and binaural cue combination. Suggestions that the binaural system may require a period of time in which to establish a baseline reference of activity in order to detect a subsequently-presented signal [19], such as within the context of the EqualizationCancellation (EC) model may also require modification for instances in which changes in noise bandwidth interact with noise duration and preceding noise segments (precursor/ fringes) [21]. 

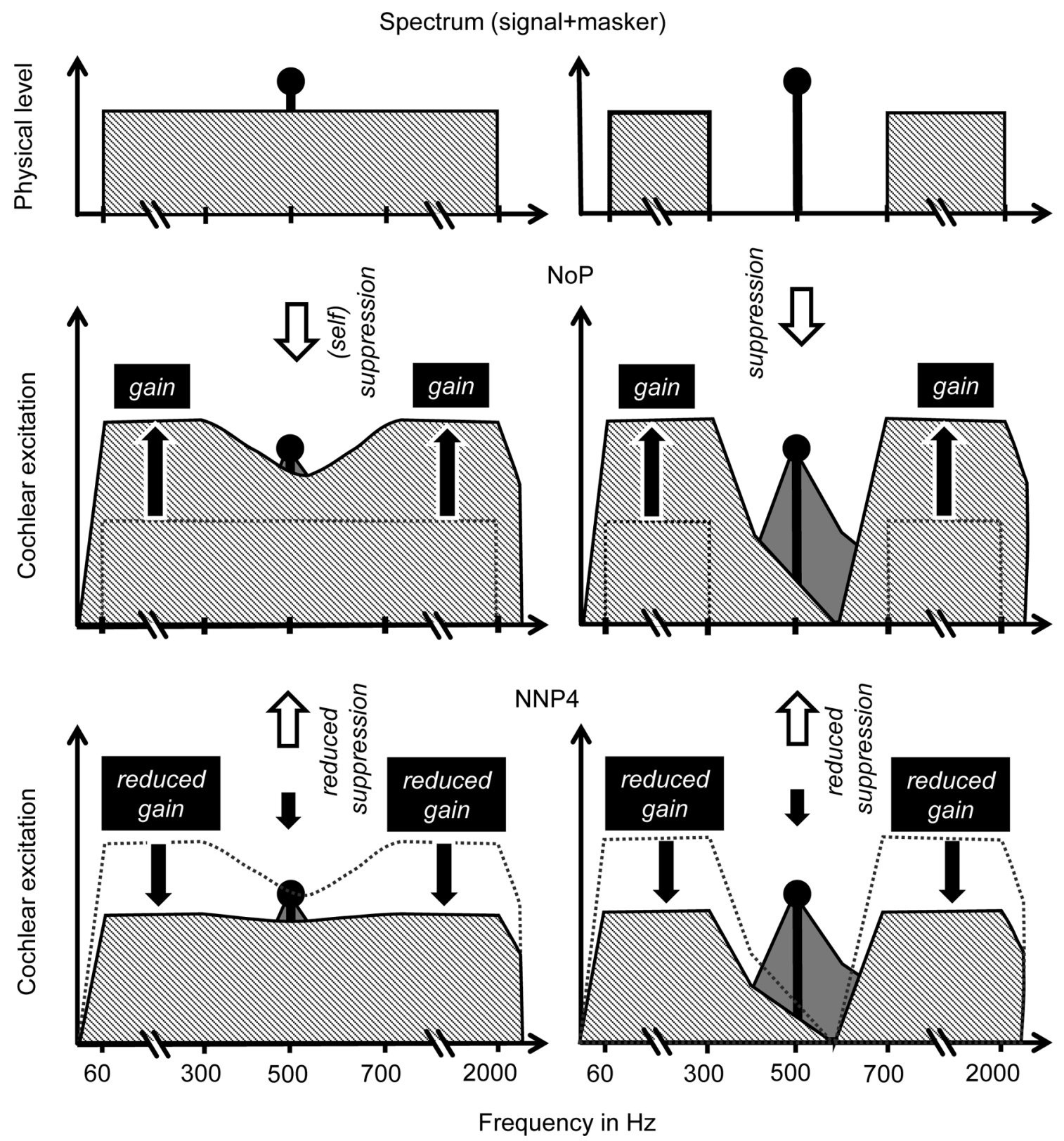

Figure 4. Explanation of the data on the basis of cochlear gain, suppression and the MOC reflex. Top row: Schematic plot of the signal and masker spectrum for the no-notch masker (left column) and the masker with a $400 \mathrm{~Hz}$ notch (right column). Masker are represented by a striped rectangles, whereas the signal in simultaneous masking paradigm by a pointed arrow. Middle row: Corresponding cochlea excitation without precursor. The excitation pattern of the masker is indicated by an area with with leftsloping stripes, whereas the excitation pattern of the signal is indicated by a dark grey area. The dashed rectangular box indicate the masker from the top row. Cochlear gain (thick black filled arrows) affects masker and signal. Due to (two-tone) suppression (thick open arrows), the simultaneously presented signal and also the middle portion of the no-notch masker (left) are suppressed. Note that the signal in the forward-masking condition is not suppressed, thus the line connecting the thresholds is steeper than in simultaneous condition. Bottom row: Cochlea excitation when precursor is added. Precursor activates MOC reflex, reducing cochlear gain. Reduction has a certain frequency specificity [67], affecting middle portion of the no-notch masker (left) less than the rest of the masker spectrum. The linearisation reduces suppressive effect within no-notch masker, leading to higher masking of nonsimultaneously presented signal. For the $400-\mathrm{Hz}$ notched-noise masker, the signal in the simultaneous condition is better audible due to reduced suppression and less gain reduction.

\section{Summary and conclusions}

Notched-noise data are commonly used to estimate the filter characteristics of the peripheral auditory filters.
By using two maskers (one with no notch, and one with a notch) the present data confirmed that introducing a notch in the masker results in a larger reduction in threshold for the diotic condition than for the dichotic condition. 
This confirms previous binaural notched noise data. The notched noise data indicate effectively wider binaural filters. The present study shows that this is not only found in a simultaneous masking condition (as used before) but also in a forward masking condition. The initial hypothesis of this study was that the MOC reflex should have a similar effect on diotic and dichotic thresholds, if the effect is only reducing cochlear gain. In general, the precursor tends to steepen the decrease in threshold as the notch width increases. The precursor effect was smaller for the $800-\mathrm{Hz}$ notched-noise precursor than for the $400-\mathrm{Hz}$ notched-noise precursor. The steepening due to the precursor can be understood on the basis of a combination of a frequency specific MOC reflex and suppression but it cannot be ruled out that other processes that were discussed in relation to the precursor effect can also account for this aspect of the data. In contrast to the initial hypothesis, diotic thresholds were more affected by the precursor than dichotic thresholds, resulting in a significantly larger decrease of the BMLD as the notch width increases, when the precursor was present. These data indicate a specific binaural aspect of the process underlying the precursor effect, since the processing of binaural cues seems to be less affected by the precursor than monaural processing. This specific binaural aspect would be of interest to study in more depth in the future.

\section{Conflict of interest}

The authors declare that they do not have any conflict of interest.

\section{Acknowledgments}

This work was supported by the Deutsche Forschungsgemeinschaft (DFG).

\section{References}

1. M.C. Liberman, S. Puria, J.J. Guinan Jr: The ipsilaterally evoked olivocochlear reflex causes rapid adaptation of the $2 \mathrm{f} 1$ - f 2 distortion product otoacoustic emission. Journal of the Acoustical Society of America 99, 6 (1996) 3572-3584.

2. I. Yasin, V. Drga, C.J. Plack, Effect of human auditory efferent feedback on cochlear gain and compression, Journal of Neuroscience 34, 46 (2014) 15319-15326.

3. J.L. Verhey, M. Kordus, V. Drga, I. Yasin, Effect of efferent activation on binaural frequency selectivity, Hearing Research 350 (2017) 152-159.

4. S.P. Bacon: Compression: from cochlea to cochlear implants, in Overview of Auditory Compression, Bacon S.P., Fay R.R., Popper A.N., Editors. Vol. 17 Springer Handbook of Auditory Research, Springer, New York, 2004, 1-17

5. J.F. Ashmore: A fast motile response in guinea-pig outer hair cells: the cellular basis of the cochlear amplifier. Journal of Physiology 388, 1 (1987) 323-347.

6. G.L. Rasmussen, Efferent fibers of the cochlear nerve and cochlear nucleus, in Neural mechanisms of the auditory and vestibular systems. G.L. Rasmussen, W.F. Windle, Editor. Vol. 8, Springfield (Ill.), 1960, pp. 105-115.
7. W.B. Warr: Olivocochlear and vestibular efferent neurons of the feline brain stem: their location, morphology and number determined by retrograde axonal transport and acetylcholinesterase histochemistry. Journal of Comparative Neurology 161, 2 (1975) 159-181.

8. I.J. Russell, E. Murugasu: Medial efferent inhibition suppresses basilar membrane responses to near characteristic frequency tones of moderate to high intensities. Journal of the Acoustical Society of America 103, 3 (1997) 1734-1738.

9. W.B. Warr, J.J. Guinan Jr: Efferent innervation of the organ of Corti: two separate systems. Brain Research 173, 1 (1979) $152-155$.

10. U.A. Kumar, C.S. Vanaja: Functioning of olivocochlear bundle and speech perception in noise. Ear and Hearing 25, 2 (2004) 142-146.

11.S.K. Mishra, M.E. Lutman: Top-down influences of the medial olivocochlear efferent system in speech perception in noise. PLoS One 9, 1 (2014) e85756.

12. I.B. Mertes, K.M. Johnson, Z.A. Dinger: Olivocochlear efferent contributions to speech-in-noise recognition across signal-to-noise ratios. Journal of the Acoustical Society of America 145, 3 (2019) 1529-1540.

13. A. Strickland: The temporal effect with notchednoise maskers: analysis in terms of input-output functions. Journal of the Acoustical Society of America 115 (2004) 2234-2245.

14.E.A. Strickland: The relationship between precursor level and the temporal effect. Journal of the Acoustical Society of America 123 (2008) 946-954.

15. D.O. Kim, P.A. Dorn, S.T. Neely, M.P. Gorga: Adaptation of distortion product otoacoustic emission in humans. Journal of the Association for Research in Otolaryngology 2, 1 (2001) 31-40.

16. B.C. Backus, J.J. Guinan: Time-course of the human medial olivocochlear reflex. Journal of the Acoustical Society of America 119, 5 (2006) 2889-2904.

17. J.J. Guinan, M.L. Gifford: Effects of electrical stimulation of efferent olivocochlear neurons on cat auditory-nerve fibers. III. Tuning curves and thresholds at CF. Hearing Research 37, 1 (1988) 29-45.

18. D.M. McFadden: Masking-level differences with continuous and with burst masking noise. Journal of the Acoustical Society of America 40 (1966) 1414-1419.

19. W.A. Yost: Prior stimulation and the masking-level difference. Journal of the Acoustical Society of America 78 (1985) 901-907.

20. R.H. Gilkey, B.D. Simpson, J.M. Weisenberger: Noise fringe and binaural detection. Journal of the Acoustical Society of America 88 (1990) 1323-1332.

21. I. Yasin, G.B. Henning: The effects of noise-bandwidth, noise-fringe duration, and temporal signal location on the binaural masking-level difference. Journal of the Acoustical Society of America 132 (2012) 327-338.

22. B. Kollmeier, R.H. Gilkey: Binaural forward and backward masking: Evidence for sluggishness in binaural detection. Journal of the Acoustical Society of America 87 (1990) 17091719.

23. M. van der Heijden, C. Trahiotis: Binaural detection as a function of interaural correlation and bandwidth of masking noise: Implications for estimates of spectral resolution. Journal of the Acoustical Society of America 103, 3 (1998) 1609-1614.

24. L.R. Bernstein, C. Trahiotis, R.L. Freyman: Binaural detection of $500-\mathrm{Hz}$ tones in broadband and in narrowband masking noise: Effects of signal/noise duration and forward masking fringes. Journal of the Acoustical Society of America 119 (2006) 2981-2993. 
25. V.M. Richards, D.E. Shub, E.M. Carreira: The role of masker fringes for the detection of coherent tone pips. Journal of the Acoustical Society of America 130 (2011) 883-892.

26. P.M. Zurek, N.I. Durlach: Noise-bandwidth dependence in homophasic and antiphasic tone detection. Journal of the Acoustical Society of America 81 (1987) 459-464.

27. S. van de Par, A. Kohlrausch: Dependence of binaural masking level differences on center frequency, noise bandwidth, and interaural parameters. Journal of the Acoustical Society of America 106 (1999) 1940-1947.

28. M.E. Evilsizer, R.H. Gilkey, C.R. Mason, H.S. Colburn, L.H. Carney: Binaural detection with narrowband and wideband reproducible noise maskers: I. Results for humans. Journal of the Acoustical Society of America 111 (2002) 336-345.

29. J. Breebaart, S. van de Par, A. Kohlrausch: Binaural processing model based on contralateral inhibition. II. Dependence on spectral parameters. Journal of the Acoustical Society of America 110 (2001) 1089-1104.

30. L.H. Carney: Binaural cues for detection of signals in noise: Experiments and models, in Proceedings of the International Symposium on Auditory and Audiological Research, Vol. 2, 2009, pp. 71-82.

31.S.G. Jennings, E.A. Strickland: Evaluating the effects of olivocochlear feedback on psychophysical measures of frequency selectivity. Journal of the Acoustical Society of America 132, 4 (2012) 2483-2496.

32. H. Fletcher: Auditory patterns. Review of Modern Physics 12, 1 (1940) 47-65.

33. E. de Boer, C.E. Bos: On the concept of the critical band, in 4th International Congress on Acoustics, Copenhagen, Denmark H13, 1962, pp. 1-4.

34. R.D. Patterson: Auditory filter shapes derived with noise stimuli. Journal of the Acoustical Society of America 59, 3 (1976) 640-654.

35. R.D. Patterson, I. Nimmo-Smith: Off-frequency listening and auditory-filter asymmetry. Journal of the Acoustical Society of America 6, 1 (1980) 229-245.

36. J.L. Verhey, S. van de Par: Binaural frequency selectivity in humans. Eurorpeen Journal of Neuroscience 51, 5 (2018) 1179-1190.

37. M.F. Yama, D.E. Robinson: Comparison of frequency selectivity for the monaural and binaural hearing systems: Evidence from a probe-frequency procedure. Journal of the Acoustical Society of America 71 (1982) 694-700.

38. M. Nitschmann, J.L. Verhey, B. Kollmeier: Monaural and binaural frequency selectivity in hearing-impaired subjects. International Journal of Audiology 49, 5 (2010) 357-367.

39. J.W. Hall, R.S. Tyler, M.A. Fernandes: Monaural and binaural auditory frequency resolution measured using bandlimited noise and notched-noise masking. Journal of the Acoustical Society of America 73, 3 (1983) 894-898.

40. M. Nitschmann, J.L. Verhey: Binaural notched-noise masking and auditory-filter shape. Journal of the Acoustical Society of America 133, 4 (2013) 2262-2271.

41. D.E. Robinson, C. Trahiotis: Effects of signal duration and masker duration on detectability under diotic and dichotic listening conditions. Attention, Perception and Psychophysics 12,4 (1972) 333-334.

42. M. Nitschmann, J.L. Verhey, B. Kollmeier: The role of across-frequency processes in dichotic listening conditions. Journal of the Acoustical Society of America 126, 6 (2009) 3188-3198.

43. A. Kohlrausch, R. Fassel: Binaural masking level differences in nonsimultaneous masking, in Binaural and spatial hearing in real and virtual environments. Lawrence Erlbaum Associates, Mahwah, NJ, 1997, pp. 169-190.
44. U.T. Zwicker, E. Zwicker: Binaural masking-level difference as a function of masker and test-signal duration. Hearing Research 13, 3 (1984) 215-219.

45. B.C.J. Moore, B.R. Glasberg: Auditory filter shapes derived in simultaneous and forward masking. Journal of the Acoustical Society of America 70, 4 (1981) 10031014.

46. M. Unoki, R. Miyauchi, C.-T. Tan: Estimates of tuning of auditory filter using simultaneous and forward notched-noise masking, in Hearing -From Sensory Processing to Perception, B. Kollmeier, V. Hohmann, G.M. Klump, U. Langemann, M. Mauermann, S. Uppenkamp, J.L. Verhey, Editors, Vol. 119, Springer-Verlag Berlin Heidelberg, Berlin, Heidelberg, 2007, pp. 19-26.

47. B.C.J. Moore: Psychophysical tuning curves measured in simultaneous and forward masking. Journal of the Acoustical Society of America 63, 2 (1978) 524-532.

48. A.J. Oxenham, C.A. Shera: Estimates of human cochlear tuning at low levels using forward and simultaneous masking. Journal of the Association for Research in Otolaryngology 4, 4 (2003) 541-554.

49. W.A. Yost, J. Walton: Hierarchy of masking-level differences optained for temporal masking. Journal of the Acoustical Society of America 61 (1977) 1376-1379.

50. M.F. Yama: Binaural analysis in forward masking: Effects of signal frequency. Journal of the Acoustical Society of America 78 (1985) 2142-2145.

51. X. Zhou, S. Henin, S.E. Thompson, G.R. Long, L.C. Parra: Sensation to masked tones following notched-noise correlates with estimates of cochlear function using distortion product otoacoustic emissions. Journal of the Acoustical Society of America 127 (2010) 970-976.

52. R.P. Carlyon: Changes in the masked thresholds of brief tones produced by preceding bursts of bandpass and notched noise. Journal of the Acoustical Society of America 83, S1 (1988) 34-34.

53. M.L. Hicks, S.P. Bacon: Factors influencing temporal effects with notched-noise maskers. Hearing Research 64, 1 (1992) $123-132$.

54. N.F. Viemeister, S.P. Bacon: Forward masking by enhanced components in harmonic complexes. Journal of the Acoustical Society of America 71 (1982) 1502-1507.

55. J.A. Beim, E. Maxwell, A.J. Oxenham, M. Wojtczak: Stimulus frequency otoacoustic emissions provide no evidence for the role of efferents in the enhancement effect. Journal of the Association for Research in Otolaryngology 16,5 (2015) 613-629.

56. L. Feng, A.J. Oxenham: Auditory enhancement and the role of spectral resolution in normal-hearing listeners and cochlear-implant users. Journal of the Acoustical Society of America 144 (2018) 552-566.

57. S.G. Jennings, E.A. Strickland, M.G. Heinz: Precursor effects on behavioral estimates of frequency selectivity and gain in forward masking. Journal of the Acoustical Society of America 125 (2009) 2172-2181.

58. H.A. Kreft, M. Wojtczak, A.J. Oxenham: Auditory enhancement under simultaneous masking in normal-hearing and hearing-impaired listeners. Journal of the Acoustical Society of America 143 (2018) 901-910.

59. N.F. Viemeister, S.P. Bacon: Forward masking by enhanced components in harmonic complexes. Journal of the Acoustical Society of America 71 (1982) 1502-1507.

60. A.J. Byrne, M.A. Stellmack, N.F. Viemeister: The enhancement effect: evidence for adaptation of inhibition using a binaural centering task. Journal of the Acoustical Society of America 129 (2011) 2088-2094. 
61. A. Almishaal, G.M. Bidelman, S.G. Jennings: Notched-noise precursors improve detection of low-frequency amplitude modulation". Journal of the Acoustical Society of America 141 (2017) 324-333.

62. M.I. Marrufo-Pérez, A. Eustaquio-Martín, E.A. LopezPoveda: Adaptation to noise in human speech recognition unrelated to the medial olivocochlear reflex. Journal of Neuroscience 38 (2018) 4138-4145.

63. M. Wojtczak, A.M. Kiamg, N.T. Torunsky: Exploring the role of medial olivococlear efferents on the detection of amplitude modulation for tones presented in noise. Journal of the Association for Research in Otolaryngology 20 (2019) 395-413.

64. S. Carcagno, C. Semal, L. Demany: No need for templates in the auditory enhancement effect. PLoS One 8, 6 (2013).

65. W. Lilaonitkul, J.J. Guinan Frequency tuning of medialolivocochlear-efferent acoustic reflexes in humans as functions of probe frequency. Journal of Neurophysiology 107, 6 (2012) 1598-1611.

66. K.N. Darrow, S.F. Maison, M.C. Liberman: Cochlear efferent feedback balances interaural sensitivity. Nature Neuroscience 9, 12 (2006) 1474-1476.

67. V. Drga, C.J. Plack, I. Yasin: Frequency tuning of the efferent effect on cochlear gain in humans. Advances in Experimental Medicine and Biology 894 (2016) 477-484.

68. B.C.J. Moore, B.R. Glasberg: Modeling binaural loudness. Journal of the Acoustical Society of America 121, 3 (2007) 1604-1612.

69. B.C.J. Moore, B.R. Glasberg, A. Varathanathan, J. Schlittenlacher: A loudness model for time-varying sounds incorporating binaural inhibition. Trends in Hearing 20 (2016) 2331216516682698.

Cite this article as: Dymel F. Kordus M. Yasin I. \& Verhey JL. 2021. A notched-noise precursor affects both diotic and dichotic notched-noise masking. Acta Acustica, 5, 43. 